\title{
Wavelet analysis of wall turbulence to study large-scale modulation of small-scales
}

\author{
W. J. Baars - K. M. Talluru • N. Hutchins · I. Marusic
}

Received: date / Accepted: date

\begin{abstract}
Wavelet analysis is employed to examine amplitude- and frequency-modulations in broadband signals. Of particular interest are the streamwise velocity fluctuations encountered in wall-bounded turbulent flows. Recent studies have shown that an important feature of the near-wall dynamics is the modulation of small-scales by large-scale motions. Small- and large-scale components of the velocity time series are constructed by employing a spectral separation scale. Wavelet analysis of the small-scale component decomposes the energy in joint time-frequency space. The concept is to construct a low-dimensional representation of the small-scale time-varying spectrum via two new time series: the instantaneous amplitude of the smallscale energy, and the instantaneous frequency. Having the latter in a time-continuous representation allows a more thorough analysis of frequency modulation. By correlating the large-scale velocity with the concurrent small-scale amplitude and frequency realizations, both amplitude- and frequency-modulations are studied. In addition, conditional averages of the small-scale amplitudeand frequency-realizations depict unique features of the scale-interaction. For both modulation phenomena, the much studied time shifts, associated with peak correlations between the large-scale velocity and small-scale amplitude and frequency traces, are addressed. We confirm that the small-scale amplitude signal leads the large-scale fluctuation close to the wall. It is revealed that the time shift in frequency modulation is smaller
\end{abstract}

W. J. Baars $(凹) \cdot$ N. Hutchins · I. Marusic

Department of Mechanical Engineering,

University of Melbourne, VIC 3010, Australia

e-mail: wbaars@unimelb.edu.au

K. M. Talluru

Discipline of Mechanical Engineering,

University of Newcastle, NSW 2308, Australia than that in amplitude modulation. The current findings are described in the context of a conceptual mechanism of the near-wall modulation phenomena.

\section{Introduction}

Continuing research on scale-interactions in turbulent boundary layers (TBLs) has revealed the modulation of small-scales by large-scale motions. These large-scale structures encompass regions of negative and positive fluctuating velocity and are extensively described in the literature (Favre et al, 1967; Blackwelder and Kovasznay, 1972; Brown and Thomas, 1977; Wark and Nagib, 1991; Ganapathisubramani et al, 2003; Tomkins and Adrian, 2003; Hutchins and Marusic, 2007a, among others). The way by which the amplitude of the large scales influences the small-scale intensity can be considered an amplitude modulation (AM) and was initially identified by Brown and Thomas (1977) and Bandyopadhyay and Hussain (1984). Over the past decade, there has been a considerable refocus on modulation and the underlying scale-interactions. It was shown by Hutchins and Marusic (2007a,b) that large-scale modulation is an important feature of high Reynolds number wall-bounded flows. Many studies followed in an attempt to fully quantify the modulation (Mathis et al, 2009; Chung and McKeon, 2010; Guala et al, 2011; Ganapathisubramani et al, 2012; Duvvuri and McKeon, 2015). Generally, in both recent and more dated studies on scale-interactions, the turbulent velocity signal has been decomposed into two sets of scales, being the large- and small-scale components; this is conceptually visualized in Fig. 1a for single-point time-resolved data. A single inner-normalized separation scale is typically taken as $\lambda_{x}^{+}=\lambda_{x} U_{\tau} / \nu=7000$ and the local mean 
(a)

$u$

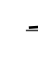

$u_{S}$

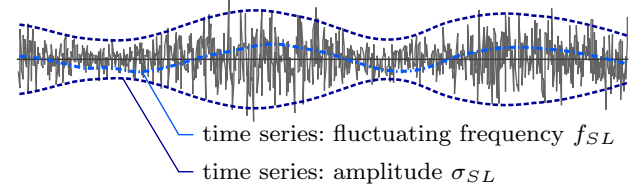

(b) Amplitude Modulation (AM): $u_{L} \& \sigma_{S L}$

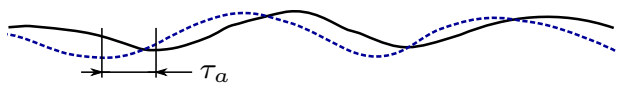

(c) Frequency Modulation (FM): $u_{L} \& f_{S L}$

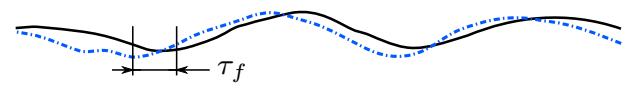

Fig. 1 a Decomposing the fluctuating velocity into large$\left(u_{L}\right)$ and small-scale $\left(u_{S}\right)$ components. b Shift in amplitude modulation and $\mathbf{c}$ frequency modulation (time increases from left-to-right); valid for the inner-region

velocity, $\bar{U}^{+}=\bar{U} / U_{\tau}$, is used in determining the separation frequency $f^{+}=\bar{U}^{+} / \lambda_{x}^{+}$(Hutchins and Marusic, 2007b; Mathis et al, 2009). Superscript ' + ' denotes the inner-scaling with friction velocity, $U_{\tau}$, and kinematic viscosity, $\nu$. The impact of large-scales on the amplitude of small-scales has been explored using averages of the small-scale intensity conditioned on positive or negative fluctuations of large-scale events (Hutchins and Marusic, 2007a; Hutchins et al, 2011). Mathis et al (2009) employed a scheme in an attempt to quantify the degree of AM, which relied on a correlation of the large-scale component with an envelope of the smallscale fluctuations, found via a Hilbert transform. The normalized correlation coefficient for a zero-time-shift, at a single location, was denoted as the AM coefficient, $R_{a}$. Bernardini and Pirozzoli (2011) considered a two-point alternative by capturing the modulating influence of an off-wall location on another location, using DNS data. Such a two-point investigation follows Marusic et al (2010), where the two-point correlation and modulation characteristics, relative to a fixed position in the logarithmic region, are determined for a predictive model of near-wall velocity fluctuations. Finally, Schlatter and Örlü (2010) showed that the AM coefficient may not be a metric to unambiguously quantify the large-scale modulation phenomenon in a time series. Nonetheless, a variety of studies provide strong evidence for the scale-interaction between large- and small-scales near the wall, dubbed as modulation.

In the 1980s, Bandyopadhyay and Hussain (1984) showed results from an almost identical AM coefficient, computed as the normalized correlation between the large-scale velocity and a pseudo-low-frequency signal created from the high-frequency component of velocity. They observed a scale-interaction that was characterized by positive correlations between large-scale velocity and small-scale intensity in the near-wall region; this occurred for a phase lead of the small-scales relative to the large-scales. Such a shift within one signal is illustrated in Fig. 1b, where the large-scale velocity is shown alongside an envelope of the small-scale fluctuations; the time shift between the solid line (largescale velocity, $u_{L}$ ) and dashed line (small-scale envelope, $\left.\sigma_{S L}\right)$ is denoted as $\tau_{a}$. Additionally, Bandyopadhyay and Hussain (1984) noticed a phase reversal within the log-region, meaning that an anti-correlation of approximately the same magnitude became dominant at an equal but opposite shift (phase lag). Others have built upon those views in an attempt to physically explain these time shifts (Chung and McKeon, 2010; Ganapathisubramani et al, 2012; Jacobi and McKeon, 2013).

In contrast to $\mathrm{AM}$, a less robust scheme exists for quantifying frequency modulation (FM), which was notably addressed for wall turbulence by Ganapathisubramani et al (2012). This work relied on a discrete technique where a count of the local extrema in the smallscale signal was representative of the dominant smallscale frequency (Rao et al, 1971; Sreenivasan et al, 1983). The number count was performed over short-time windows of the time series and their characteristics were binned according to the amplitude and sign of the largescale fluctuations. Large-scale conditional views of smallscale frequency revealed that FM was absent for wallnormal locations above $\approx 100$ wall units at $R e_{\tau}=$ $U_{\tau} \delta / \nu=14150$, where $\delta$ is the boundary layer thickness. Additionally, Ganapathisubramani et al (2012) showed that FM encompasses a time shift between largeand small-scale fluctuations that is qualitatively similar to that in AM near the wall; this shift is denoted here as $\tau_{f}$ and illustrated in Fig. 1c. Their discrete technique was shown to work well for exploring how the strength of large-scales affects the modulation, however, the shorttime windowing technique is less applicable when time shifts in AM $\left(\tau_{a}\right)$ and FM $\left(\tau_{f}\right)$ are to be investigated.

The aim of the present work is to employ a scheme that quantifies magnitude and phase information of AM and FM embedded in broadband time series acquired in a TBL (Sect. 2). The scheme is suitable for investigating how a range of low frequencies modulate - or interact with - a range of higher frequencies. We apply wavelet analysis in Sect. 3.1 to construct two new time series, in what is conceptually a low-dimensional approach. That is, two time series will reflect the time-varying smallscale energy in terms of its instantaneous amplitude and 


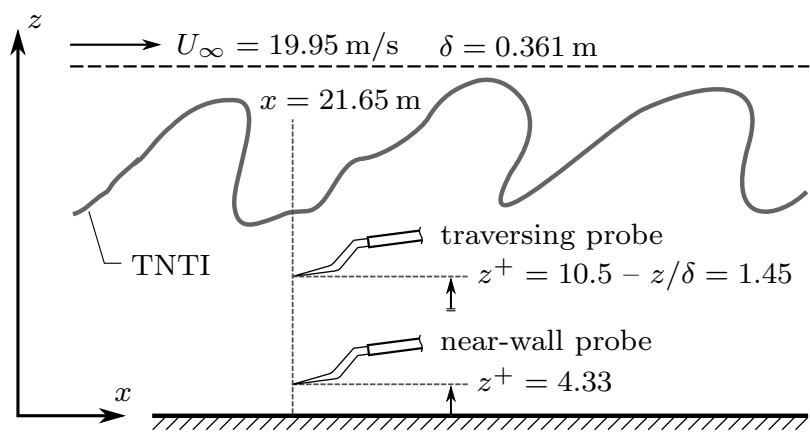

Fig. 2 Experimental arrangement of the two-probe hotwire measurements in Melbourne's boundary layer facility

mean frequency. Modulation-type interactions between large- and small-scales are then investigated in Sect. 4. The type of modulations that could be analyzed are not limited to turbulent flows and are present in many research areas, such as seismology (Neuberg, 2000) and wind turbine acoustics (Larsson and Öhlund, 2014)

\section{Experiments}

\subsection{Hardware and instrumentation}

Experiments were carried out in the high Reynolds number boundary layer facility at the University of Melbourne (Nickels et al, 2005). The tunnel has a test section length of $27 \mathrm{~m}$, which ensures that high spatial and temporal resolution can be obtained within a high Reynolds number boundary layer using existing instrumentation under moderate free stream velocities. The pressure coefficient $C_{p}$ was maintained constant to within $\pm 0.87 \%$ (Kulandaivelu, 2011). Free-stream turbulence intensities are nominally less than $0.05 \%$ at the test section inlet.

The data employed in the current study corresponds to two hotwire probes with an arrangement shown in Fig. 2. The near-wall probe is a boundary layer Dantec 55P30 probe with a Platinum-Wollaston wire of $d=5 \mu \mathrm{m}$ diameter, while the traversing hotwire consists of a Dantec $55 \mathrm{P} 15$ probe with a $d=2.5 \mu \mathrm{m} \mathrm{Pt}$ wire. The near-wall and traversing sensors are etched to a length of $l=1.0 \mathrm{~mm}$ and $l=0.5 \mathrm{~mm}$, respectively, to maintain a $l / d$ ratio of 200 , as recommended by Ligrani and Bradshaw (1987). The near wall probe is positioned at $0.105 \mathrm{~mm}$ from the wall while the traversing probe is moved to 40 logarithmically spaced points in the range $0.25 \mathrm{~mm} \leq z \leq 525 \mathrm{~mm}$. The wall at this measurement location is made of glass to reduce heat conduction from the hotwire to the wall.

Both hotwire probes are operated in constant temperature mode, with an overheat ratio of 1.8 , using an in-house built anemometer (MUCTA). For each measurement point, time series from both probes were acquired simultaneously for a duration of 300 seconds at a sampling rate of $20 \mathrm{kHz}$ using a 16-bit Speedgoat IO106 module embedded in a Speedgoat Performance realtime target machine. Before A/D conversion, the signals were filtered using a $4^{\text {th }}$-order Butterworth filter with a cut-off at $10 \mathrm{kHz}$. The traversing hotwire probe was calibrated against a Pitot-static tube before and after the experiment at 12 different free stream velocities, $U_{\infty}$, ranging from $0 \mathrm{~m} / \mathrm{s}$ to $23 \mathrm{~m} / \mathrm{s}$. In addition, the traversing probe was moved to the free stream location after every five measurement points in the boundary layer and the voltage of the hotwire in the free stream flow was logged to correct for hotwire drift, see Talluru et al (2014b) for a full description of the method. An indirect method of calibration (Chauhan et al, 2009) was used for the inner hotwire probe which was simultaneously calibrated. At each of the 12 calibration speeds, corresponding mean voltages were recorded for the inner-probe that was situated within the linear region $\left(U^{+}=z^{+}\right)$of the mean velocity profile (Sect. 2.2). Note that the inner-normalized position of the probe changes with free stream velocity, as $z^{+}=z U_{\tau} / \nu$, and $U_{\tau}$ is a function of $U_{\infty}$. Here, we adopted an empirical fit between $U_{\tau}$ and $U_{\infty}$ as provided by Chauhan et al (2009).

\subsection{Flow conditions and bounday layer statistics}

Measurements were made at a streamwise distance of $x=21.65 \mathrm{~m}$ from the trip at the test section inlet $(x=0)$, with a free stream velocity of nominally $20 \mathrm{~m} / \mathrm{s}$. The boundary layer at this location has a thickness of $0.361 \mathrm{~m}$ and the friction velocity is $U_{\tau}=0.626 \mathrm{~m} / \mathrm{s}$. These parameters were obtained by fitting the mean velocity profile to the composite profile of Chauhan et al (2009) with log-law constants $\kappa=0.384$ and $A$ $=4.17$. On the basis of these values, the Kármán number is $R e_{\tau}=14750$ and the Reynolds number based on momentum thickness is $\operatorname{Re}_{\theta}=U_{\infty} \theta / \nu=41100$. The boundary layer characteristics are listed in Table 1. Given these conditions, the sensing element of the traversing hotwire has a viscous scaled length of $l^{+}=$ $l U_{\tau} / \nu \approx 22$ and is of an acceptable length for TBL measurements. The near-wall hotwire has a length of $l^{+} \approx$ 41 , which causes an attenuation of the energy levels at wavelengths smaller than the separation scale employed in our study $\left(\lambda_{x}^{+}=7000\right.$, following Hutchins et al, 2009). Nevertheless, the attenuation does not adversely affect our results since only the large-scale content of the near-wall wire is utilized. The non-dimensional acquisition rate was $\Delta T^{+} \approx 1.3$ and guaranteed the ab- 
Table 1 Experimental parameters of the two-point hotwire study at Melbourne's boundary layer facility

\begin{tabular}{ll|ccc}
\hline boundary layer & $\begin{array}{c}\text { hotwire acquisition } \\
\text { near-wall } \\
(\mathrm{d}=5 \mu \mathrm{m})\end{array}$ & $\begin{array}{c}\text { traversing } \\
(\mathrm{d}=2.5 \mu \mathrm{m})\end{array}$ \\
\hline$x$ & $21.65 \mathrm{~m}$ & $l^{+}$ & 40.8 & 21.6 \\
$U_{\infty}$ & $19.95 \mathrm{~m} / \mathrm{s}$ & $l / d$ & 200 & 212 \\
$\delta$ & $0.361 \mathrm{~m}$ & $\Delta T^{+}$ & & 1.28 \\
$\theta$ & $0.0316 \mathrm{~m}$ & & & \\
$U_{\tau}$ & $0.626 \mathrm{~m} / \mathrm{s}$ & $T U_{\infty} / \delta$ & & 20000 \\
$\nu / U_{\tau}$ & $24.49 \mu \mathrm{m}$ & $z_{\min }^{+}$ & 4.33 & 10.5 \\
$R e_{\tau}$ & 14750 & $z_{\max } / \delta$ & - & 1.45 \\
$R e_{\theta}$ & 41100 & & & \\
\hline
\end{tabular}

sence of temporal attenuation (Hutchins et al, 2009). At each location, a long acquisition time of approximately $T U_{\infty} / \delta=20000$ was chosen for obtaining converged spectral statistics at the longest energetic wavelengths (Mathis et al, 2009). Parameters for the hotwire acquisition are listed in Table 1.

Figure 3a shows the boundary layer profiles of the mean velocity and turbulence intensity. The position of the near-wall wire is shown with the dash-dot line. One can notice a peak in the turbulence intensity profile at $z^{+} \approx 12$, which relates to the inner-spectral peak in the contour map of the pre-multiplied energy spectra shown in Fig. 3b. Note that the local mean velocity, $\bar{U}$, is used when transforming frequency to wavelength, $\lambda_{x}=\bar{U} / f$. The inner-spectral peak resides at a wavelength of $\lambda_{x}^{+} \approx$ 1000 while an outer-spectral peak appears in the logregion $\left(z^{+} \approx 3.9 R e_{\tau}^{1 / 2}=474\right)$; this is consistent with earlier work in the same facility (Mathis et al, 2009).

The two-probe data allows us to investigate the correlation of the large-scale structures relative to the nearwall probe location. The coherence aspect is often visualized using conditional averages or two-point correlation maps (e.g. Hutchins et al, 2011, and the references therein). The two-point correlation map is shown here in Fig. 4, and is computed for the long-wavelength pass-filtered fluctuating velocity signals of the nearwall and traversing probes, and are denoted as $u_{L}$ and $\tilde{u}_{L}$, respectively; a spectral filter with a pass-band of $\lambda_{x}^{+}>7000$ was used. The normalized cross-correlation coefficient, given by Eq. (1), is the inverse Fourier transform of cross-spectrum $G_{u_{L} \tilde{u}_{L}}(f)=\mathcal{F}\left[u_{L}\right]\left(\mathcal{F}\left[\tilde{u}_{L}\right]\right)^{*}$. Here, $\mathcal{F}$ denotes the Fourier transform and superscript * indicates the complex conjugate.

$\rho_{u_{L} \tilde{u}_{L}}(\tau, z)=\frac{\mathcal{F}^{-1}\left[G_{u_{L} \tilde{u}_{L}}\left(\lambda_{x} ; z\right)\right]}{\sqrt{\overline{u^{2}}} \sqrt{\overline{\tilde{u}^{2}}}}$,

The two-point correlation map seemingly illustrates the inclination of large scales as $\rho_{u_{L} \tilde{u}_{L}}(\tau, z)$ includes coupled amplitude and phase information. The time shift associated with the peak correlation, at each wall-normal
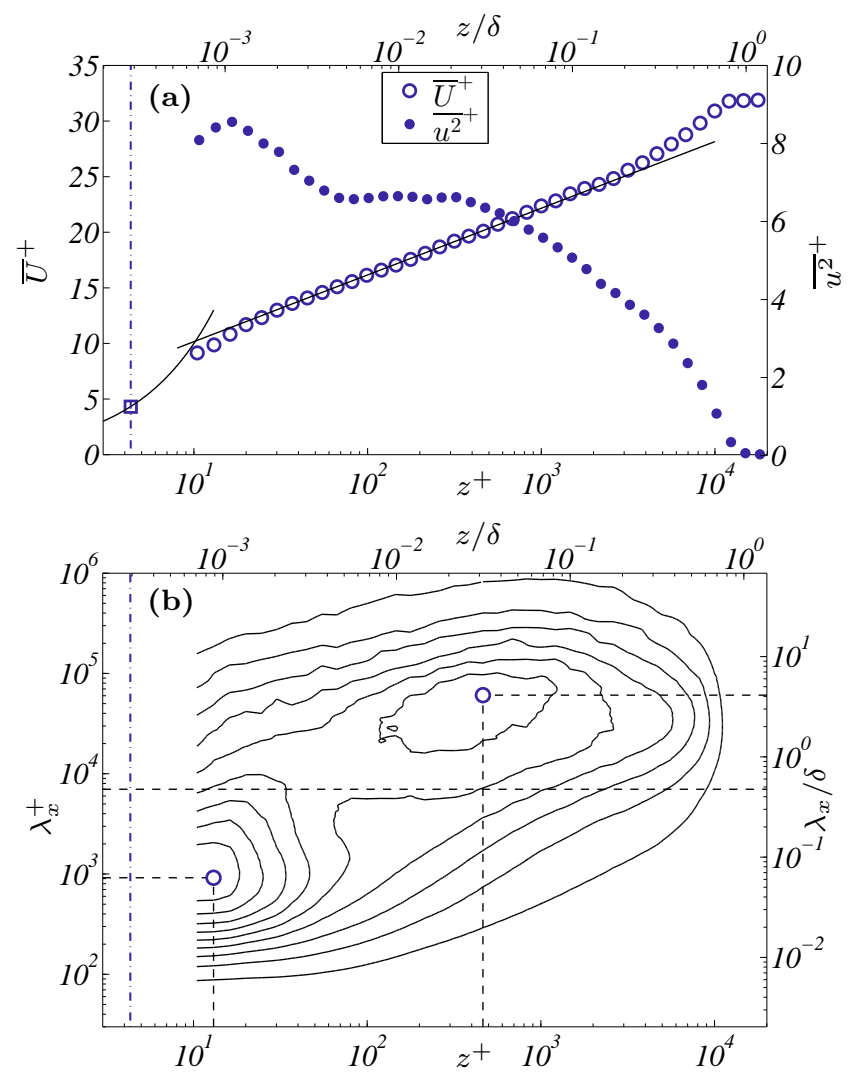

Fig. 3 a Profile of mean velocity and turbulence intensity of the streamwise velocity component at $R e_{\tau}=14750$. b Pre-multiplied energy spectra of the streamwise velocity fluctuations $k_{x} \phi_{u u} / U_{\tau}^{2}$ (level range $0.2-1.8$, level step 0.2 ); the position of the near-wall wire is shown with the dash-dot line

position, is identified as $\tau_{L}$ and visualized by the circles. The convention is that a negative shift $(\tau<0)$ implies a lag of the wall-wire signal relative to the time series of the traversing probe. Time shifts are often encountered in terms of a physical inclination angle (Marusic and Heuer, 2007), which can be obtained by the transformation $\theta=\tan ^{-1}\left[\mathcal{D} /\left(u_{c} \tau\right)\right]$, where $\mathcal{D}$ is the separation distance between the wires and $u_{c}$ is a convective speed, often chosen as the mean velocity at the location of the outer-spectral peak. Note that we will present time shifts in this work, as opposed to inclination angles, since this avoids the burden of having to choose a convective speed. Additionally, any small streamwise misalignment of the two wires can significantly impact the value of $\theta_{L}$ for small wire-separation distances, while an inaccurate placement (within $0.5 \mathrm{~mm}$ ) results in errors less than five viscous time scales when the mean velocity of the wall-probe is employed. 


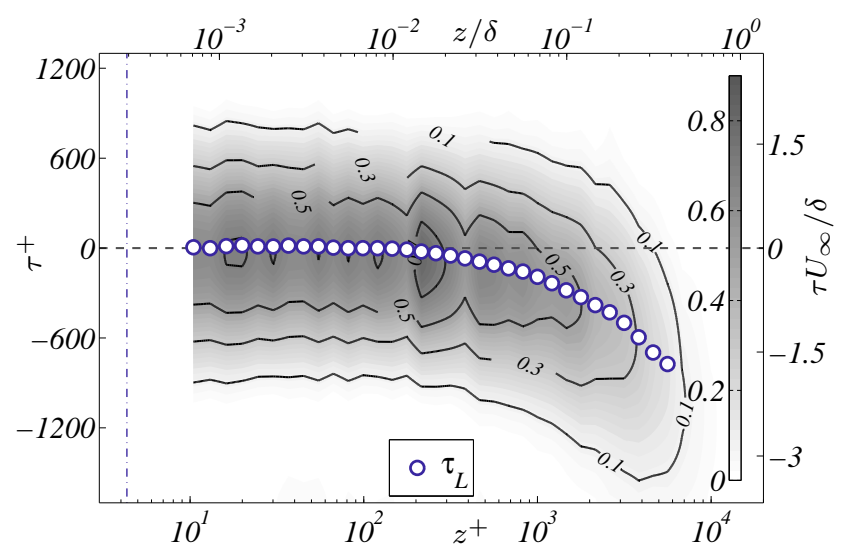

Fig. 4 Isocorrelation map of the large-scale streamwise velocity fluctuations relative to the near-wall probe location indicated with the dash-dot line. Peak locations in the isocorrelation map, $\tau_{L}$, at each wall-normal position, are visualized by the circles and represent the physical inclination of the coherent large-scale structures

\section{Extracting modulation phenomena}

As reviewed in Sect. 1, it continues to be a challenge to uniquely quantify $\mathrm{AM}$ and FM embedded within a broadband signal. Hence, we tackle this by applying the wavelet analysis (Sect. 3.1), followed by a lowdimensional representation of small-scale energy (Sect. 3.2) The concept is to generate two new time series that constitute the large-scale, time-varying, small-scale energy in terms of its instantaneous magnitude and mean frequency.

\subsection{Wavelet analysis}

The general aim of joint time-frequency analysis is to preserve the temporal information embedded in a time series, while simultaneously assessing its spectral properties (Cohen, 1989; Farge, 1992). We consider the class of wavelet analyses for computing the spectral energy density of the fluctuating streamwise velocity signal, $u$, as a function of time, in a similar fashion as presented by Baars and Tinney (2013). Wavelet transforms constitute convolutions of a mother wavelet, $\psi$, with velocity signal $u$. The wavelet forms the basis function of the transform and is analogous to harmonic functions employed in Fourier analysis. Two wavelets are used, the Morlet and Mexican hat wavelets, which are given by the following two expressions, respectively.

$$
\begin{aligned}
& \psi\left(t / t_{s}\right)=e^{j \omega_{\psi} t / t_{s}} e^{-\left|t / t_{s}\right|^{2} / 2}, \\
& \psi\left(t / t_{s}\right)=\left(1-\left|t / t_{s}\right|^{2}\right) e^{-\left|t / t_{s}\right|^{2} / 2} .
\end{aligned}
$$

The complex-valued Morlet wavelet is a harmonic wave (non-dimensional frequency taken as $\left|\omega_{\psi}\right|=6$ ) modulated by a Gaussian function, whereas the Mexican hat wavelet is the $2^{\text {nd }}$ derivative of a Gaussian. The arguments in Eq. (2) are the temporal coordinate, $t$, and the wavelet time scale, denoted as $t_{s}$. From the mathematical intricacies of wavelet transforms (e.g. Farge, 1992; Addison, 2002) it follows that an inherent tradeoff between the spectral and temporal resolutions of the transform outcome is inevitable, which is illustrated at the end of this section. Regarding the transform, complex wavelet coefficients are generated by the convolution

$\tilde{u}\left(z^{+} ; t_{s}, t\right)=\int u\left(z^{+}, t^{\prime}\right) \psi^{*}\left(\frac{t^{\prime}-t}{t_{s}}\right) d t^{\prime}$,

where superscript $*$ indicates the complex conjugate. The convolution is performed in the frequency domain and is repeated for various wavelet time scales $t_{s}$, so that the signal is decomposed in time-frequency space. The energy density, in the form of a time-varying frequency spectrum, is now obtained by taking the squared modulus of the coefficients, via

$E\left(z^{+} ; t_{s}, t\right)=\frac{\left|\tilde{u}\left(z^{+} ; t_{s}, t\right)\right|^{2}}{t_{s}}$,

). and scale argument $t_{s}$ is transformed to an equivalent frequency, $f$. Time-varying spectra are presented in the current work in the form of the one-sided Wavelet Power Spectrum (WPS), given by $E\left(z^{+} ; f, t\right)$, and defined for positive frequencies only $(f \geqslant 0)$. The wavelet transforms comprise 105 logarithmically spaced scales to resolve frequencies ranging from $5 \mathrm{~Hz}$ to $f_{s} / 2$ and ensures that all small-scales $\left(\lambda_{x}^{+}<7000\right)$ are well-captured. The WPS is illustrated by considering a short snapshot of the velocity time series (Fig. 5b) acquired by the traversing wire at $z^{+}=10.5$. The associated WPS is shown in Fig. 5a and is computed using the Mexican hat wavelet; the contour reflects the pre-multiplied energy spectrum $k_{x} \phi_{u u} / \overline{U^{2}}$ and equals $\bar{E} f / \overline{U^{2}}$.

We now accentuate a few characteristics of the WPS. Since wavelets are mutually similar among scales, the temporal resolution of the WPS is lower at larger scales. This is illustrated in Fig. 5a by a so-called cone-ofinfluence (COI) shown at the arbitrary time instant $t_{c}^{+}=10000$. The COI indicates the temporal span of the time series that influences the local WPS at time $t_{c}$, as a function of scale. In practice, we transform a time series partition of $N=2^{14}$ samples to obtain its WPS. The finite time spanned by wavelets inherently causes the WPS to be affected at the start and end of the signal, for a duration equal to the half-width of the COI. We therefore extract the unaffected region, for the resolved frequency range $\left(5 \mathrm{~Hz}<f<f_{s} / 2\right)$, and repeat 

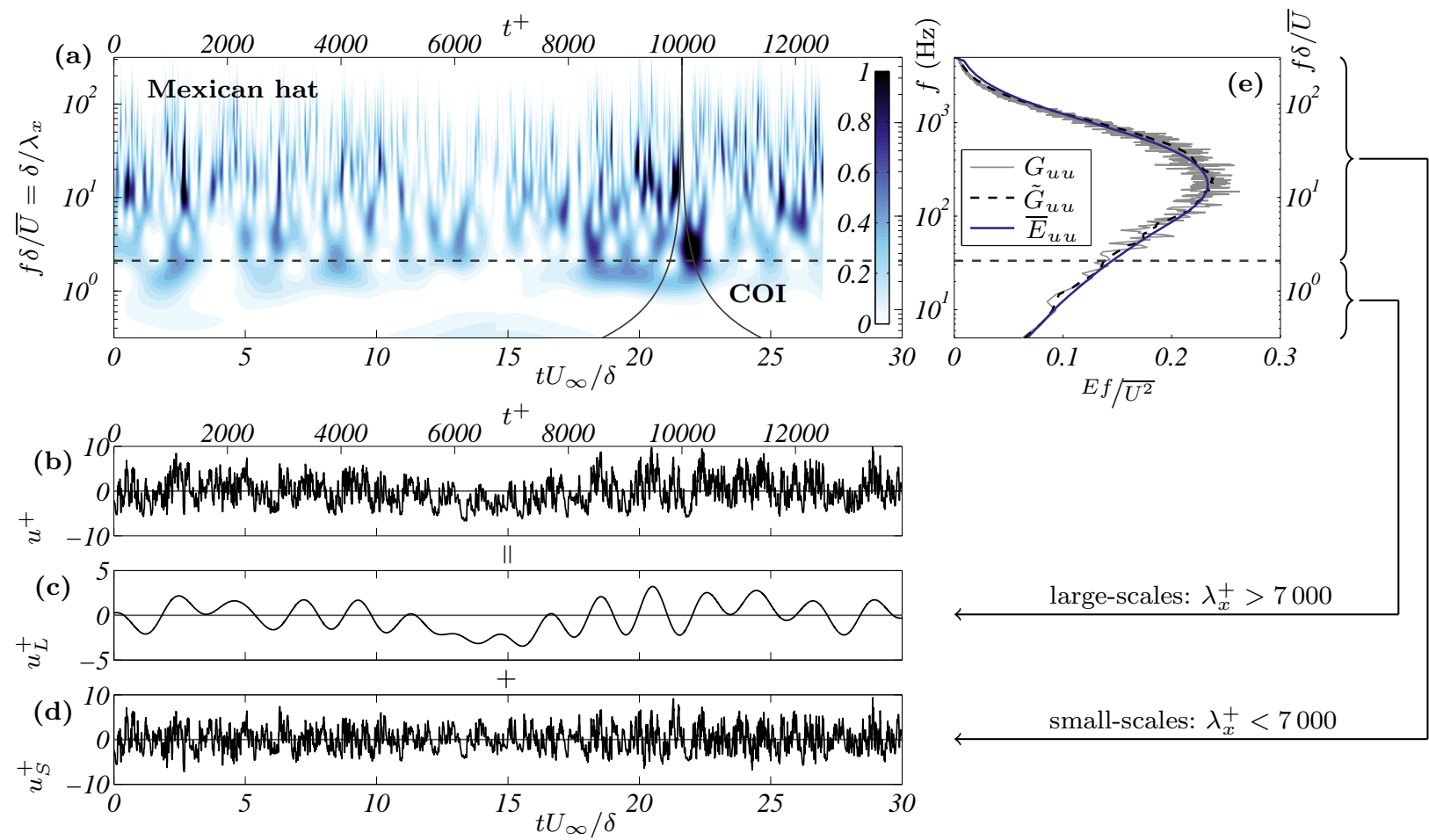

Fig. 5 a Pre-multiplied Mexican hat Wavelet Power Spectrum (WPS) of the streamwise velocity signal at $z^{+}=10.5$, shown in sub-figure $\mathbf{b}$; contour represents $k_{x} \phi_{u u} \overline{U^{2}}$. c, $\mathbf{d}$ The large- $\left(u_{L}\right)$ and small-scale $\left(u_{S}\right)$ components of the streamwise velocity fluctuations. e Comparison of the global WPS $\left(\bar{E}_{u u}\right)$ and conventional Fourier spectrum $\left(G_{u u}\right.$ : unfiltered, and $\tilde{G}_{u u}$ : filtered with a bandwidth moving average filter of $20 \%$ )

the transform for overlapping partitions of the time series to obtain a continuous WPS. The resulting WPS is invalid at just the start and end of the time series; those parts are discarded in our analysis and constitute a total of less than $0.6 \mathrm{~s}$ of data (note that $t=0$ in Fig. 5 does not correspond to the start of the acquisition). Time-averaging the WPS returns the global one-sided WPS according to $\bar{E}=1 / T \int_{0}^{T} E d t$, where $T$ is the time span of the WPS. In Fig. 5e we compare the global WPS to the unfiltered $\left(G_{u u}\right)$ and filtered $\left(\tilde{G}_{u u}\right)$ one-sided Fourier spectra. Visual inspection of Fig. 5a reveals that joint time-frequency representations of energy reveal instants of high energy at certain scales, e.g. the signal is strongly correlated with the wavelet. The energy magnitude associated with such intermittent events (Farge, 1992) can exceed 1.0 on this scale, which is more than four times the maximum $(0.23)$ in the global spectrum. For the remainder of Fig. 5, subfigures $\mathbf{c}$ and $\mathbf{d}$, we consider the scale decomposition. Here we decompose the spectral energy in two sets of scales, driven by the physical knowledge of having an inner- and outer-spectral peak in the boundary layer spectogram at this high Reynolds number (Hutchins and Marusic, 2007b). We employ the regularly chosen separation scale of $\lambda_{x}^{+}=7000$ (thus frequency scale $\left.f^{+}=\bar{U}^{+} / \lambda_{x}^{+}\right)$which is indicated by the horizontal dashed line in Figs. 5a and 5e; note that a smooth roll-off-using half the span of a hanning window - is employed over five discrete frequency scales in practice. The large- $\left(u_{L}\right)$ and small-scale $\left(u_{S}\right)$ components of velocity are shown in Figs. 5c and 5d, respectively. Mathis et al (2009) showed that their results for AM were only weakly dependent on the chosen separation scale. Similarly, our principal conclusions made later on are unaffected by the choice of separation scale, which was confirmed by investigating separation wavelengths in the range $3500<\lambda_{x}^{+}<14000$.

The resolution trade-off in the joint time-frequency distribution, as affected by the choice of wavelet, is now discussed. Each wavelet is characterized by a normalized time scale, $t / t_{s}$, and frequency scale, $t_{s} \omega$. The former governs the finite time spanned by the wavelet, while the energetic spectral scales of the Fourier transform of the wavelet span a finite domain in $t_{s} \omega$ space. A high temporal resolution is achieved when the time scale is short. Consequentially, this results in a broader frequency content centered around the characteristic frequency of the wavelet. Therefore, a high temporal resolution is associated with an intrinsic low frequency resolution; this is known as the natural scale filtering of wavelet transforms (Daubechies, 1992; Farge, 1992). For the Mexican hat wavelet, a relatively high temporal 
resolution (narrow COI) is achieved with a lower resolution in frequency as compared to other wavelets; this is evident from the vertically elongated features in the contour (Fig. 5a). In practice, different mother wavelets are employed to gain a high resolution in either time or frequency, depending on the application. In comparison to the Mexican hat wavelet, the Morlet wavelet has an improved frequency resolution, while the multitude of temporal wavelet oscillations results in a lower resolution in time. This becomes evident when comparing the Morlet WPS (Fig. 6a), for the same snapshot of the velocity signal, to the Mexican hat WPS (Fig. 5a).

\subsection{Constructing small-scale amplitude and frequency}

Concerning the amplitude of small-scale fluctuations, a well-accepted procedure in the field of wall-bounded flows (Mathis et al, 2009) is to take a Hilbert transform of the small-scale velocity, $u_{S}$, to construct an envelope of the fluctuations, $E\left(u_{S}\right)$. Subsequently, the envelope is pass-filtered at $\lambda_{x}^{+}>7000$ to obtain large-scale amplitude variations of small-scales, known as $E_{L}\left(u_{S}\right)$. We employ the WPS to construct a similar signal for the large-scale variation of small-scale amplitude; the procedure is visualized in Fig. 6. Here, Figs. 6a-d and 6g, are identical to Fig. 5, except that the Morlet WPS is shown as opposed to the Mexican hat WPS. When extracting the local spectrum at random time instant $t_{i}$, we retrieve Fig. 6h. Moving forward in this diagram, we only consider the small-scale energy of the WPS and compute the energy contained in these scales via Parseval's theorem, according to

$\sigma_{S}\left(t_{i}\right)=\left[\int_{f_{c}}^{f_{N}} E\left(f, t_{i}\right) d f\right]^{1 / 2}$.

Here, lower bound $f_{c}$ corresponds to the separation scale and upper bound, $f_{N}=f_{s} / 2$, is the Nyquist frequency. The square root of the integral reflects an instantaneous standard deviation, hence, we denote this quantity as $\sigma_{S}\left(t_{i}\right)$. The instantaneous standard deviation, for all time instants, has a non-zero-mean, and can therefore be written in terms of a fluctuating component, following $\sigma_{S}\left(t_{i}\right)=\sqrt{\overline{u_{S}^{2}}}+\sigma_{S}^{\prime}\left(t_{i}\right)$. The magnitude of $\sigma_{S}$ at instant $t_{i}$ is visualized by the horizontal rectangle in Fig. 6h. To obtain the large-scale variation of small-scale amplitude we compute Eq. (5) for all times, followed by a long-wavelength pass-filter $\left(\lambda_{x}^{+}>7000\right)$ to obtain $\sigma_{S L}(t)$; Fig. 6e presents both time series, $\sigma_{S}^{\prime}(t)$ and $\sigma_{S L}^{\prime}(t)$, normalized by friction velocity $U_{\tau}$. Long-wavelength pass-filtering ensures that we focus on the large-scale variation of the small-scale amplitude fluctuations so that linear correlations with the large-scale velocity, $u_{L}$, are meaningful (Sect. 4).

The new time series of small-scale amplitude variations, $\sigma_{S L}(t)$, could have been established using other procedures. Nevertheless, the strength of our scheme becomes evident when the local frequency content is considered. For that, a second time trace is constructed of the time-varying small-scale frequency, which is more widely known in the signal processing literature as the instantaneous frequency (IF); see reviews of Boashash (1992) and Cohen (1995). We compute the IF as the first spectral moment of the instantaneous WPS,

$f_{S}\left(t_{i}\right)=10^{f^{*}\left(t_{i}\right)}$, where

$f^{*}\left(t_{i}\right)=\frac{1}{\left[\sigma_{S}\left(t_{i}\right)\right]^{1 / 2}} \int_{f_{c}}^{f_{N}} E\left(f, t_{i}\right) f \log _{10} f d \log _{10} f$.

The IF has a non-zero mean and is presented in terms of the decomposition, $f_{S}\left(t_{i}\right)=\bar{f}_{S}+f_{S}^{\prime}$ with units of $\mathrm{s}^{-1}$; the prime notation denotes the zero-mean IF fluctuation. The IF, illustrated by the vertical rectangle in Fig. 6h, visualizes the geometric center of the local small-scale WPS, e.g. half the small-scale energy resides at $f<f_{S}^{\prime}$. Analogous to computing amplitude signal $\sigma_{S L}(t)$, Eq. (6) is evaluated for all time instants and long-wavelength pass-filtered to obtain $f_{S L}^{\prime}(t)$, shown in Fig. 6f. For instants when $f_{S L}^{\prime}>0$, the resultant spectral energy of the small-scales resides at higher frequencies than their mean frequency; vice versa for $f_{S L}^{\prime}<$ 0 . It is important to realize how our study of FM is different than that of Ganapathisubramani et al (2012). They considered the local number of extrema in the small-scale velocity signal to construct a representative frequency. This procedure is known to produce a representative frequency in terms of the Taylor microscale, $\bar{U} / \lambda_{T}(t)$ (Sreenivasan et al, 1983). The Taylor microscale, $\lambda_{T}$ is physically interpreted as the internal viscous shear-layer thickness (Dimotakis, 2005). We consider FM in terms of the $I F, f_{S}(t)$, which is physically interpreted as the local mean frequency of the small-scales; thus half of the small-scale energy resides at frequencies above and below this IF.

Before continuing we will comment on how the choice of the wavelet affects the procedure of extracting the instantaneous frequency signal, $f_{S L}^{\prime}(t)$. It became clear in Sect. 3.1 that the Morlet wavelet-based WPS constitutes a higher resolution along the frequency dimension. This is re-illustrated in Fig. 7, where the local Morlet WPS (duplicated from Fig. 6h) is compared with the local Mexican hat WPS at the same time instant. It is clear that the Mexican hat wavelet has a higher degree of natural scale filtering. Since we compute the 


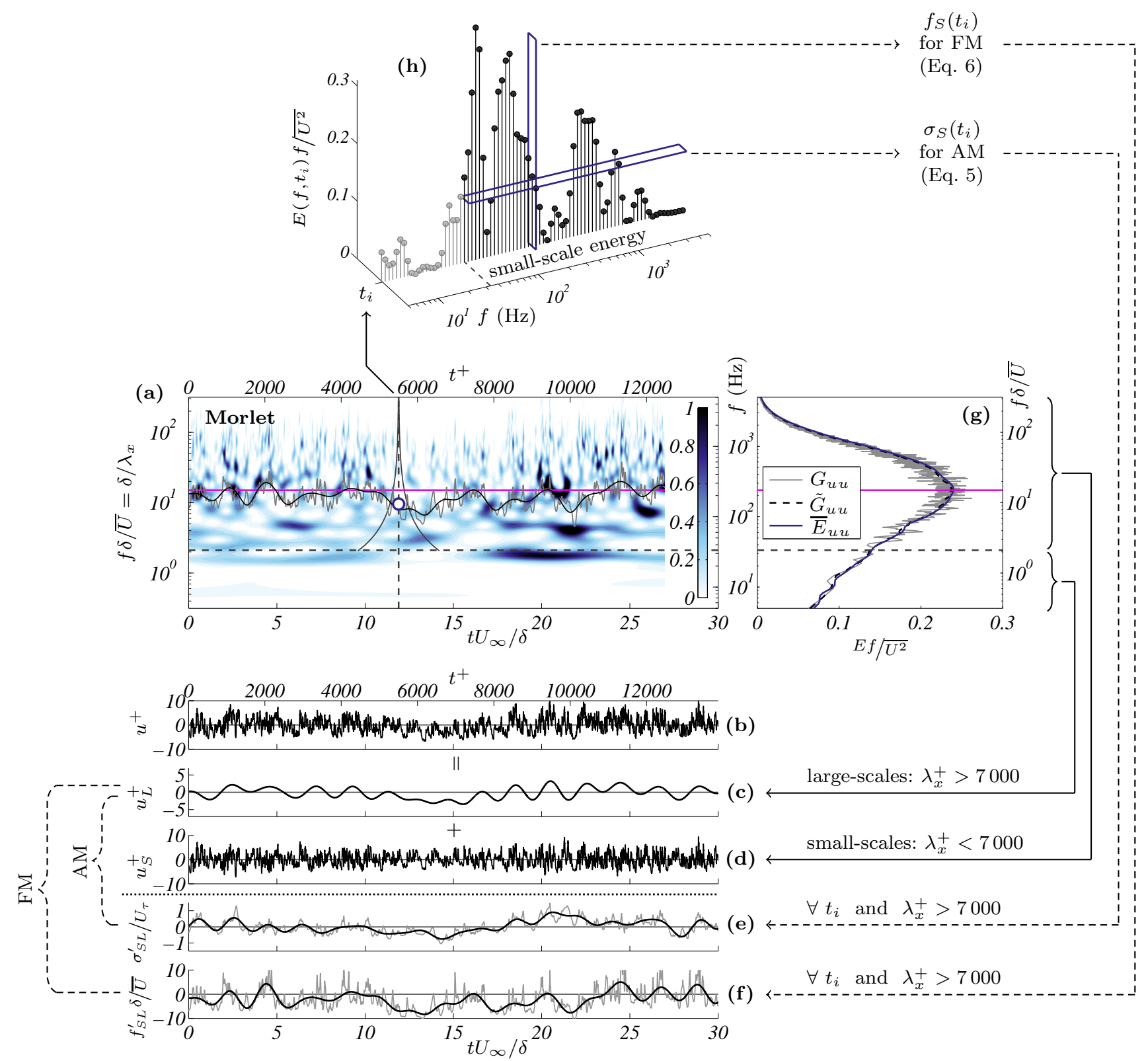

Fig. 6 a,b,c,d,g Identical to Fig. 5, but sub-figures a,b exhibit the pre-multiplied Morlet WPS. h Local Morlet WPS at time-instant $t_{i}$; the horizontal and vertical rectangles illustrate the instantaneous energy and mean frequency of the smallscales, respectively. e,f Time series of fluctuating small-scale amplitude $\sigma_{S L}^{\prime}(t)$ and instantaneous frequency $f_{S L}^{\prime}\left(t_{i}\right)$; grey lines are unfiltered signals, while black lines are the long-wavelength pass-filtered signals. Regarding the IF signal, its mean $\left(\bar{f}_{S}\right.$, magenta line) is superposed on sub-figures $\mathbf{a}$ and $\mathbf{g}$, and the time series of IF (sub-figure $\mathbf{f}$ ) is re-shown in sub-figure $\mathbf{a}$

first spectral moment of the instantaneous WPS for determining the instantaneous frequency (Eq. 6), and given that a better resolved frequency spectrum aids in a more accurate determination of the moment, it may be argued that the Morlet wavelet is more suitable in this regard. On the contrary, its different (lower) resolution in time does induce a limit in resolving all temporal fluctuations. However, the effect of a lower temporal resolution may not propagate in the results given our interest in the large-scale variation of the instantaneous small-scale amplitude and frequency content. Nevertheless, because of the inevitable trade-off in time/frequency resolution, we present results of our modulation analysis (Sect. 4) by using both Morlet and Mexican hat wavelets.

The procedure described above resulted in two new time series for each original time trace of streamwise velocity, $u(t)$. That is, we obtained $\sigma_{S L}^{\prime}(t)$ with units 


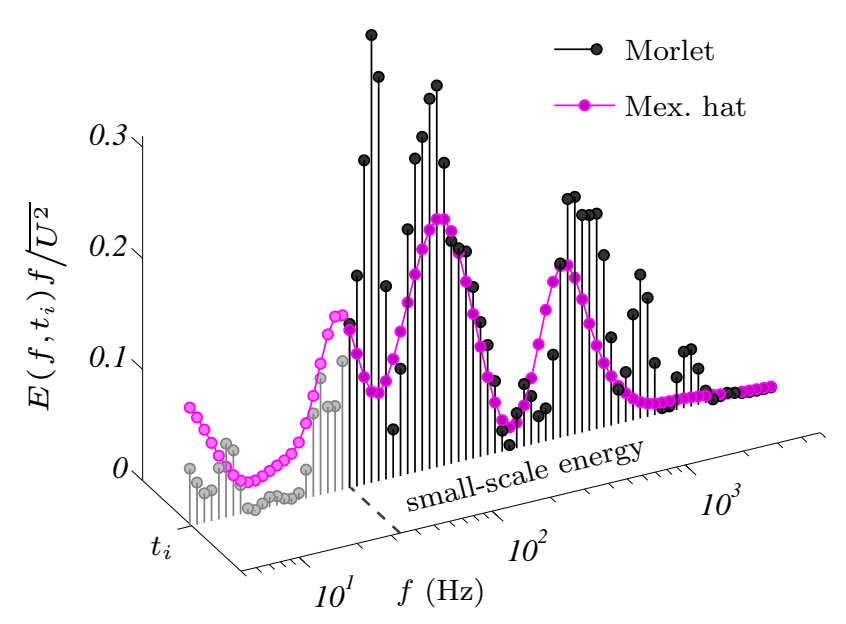

Fig. 7 Comparison of the local Morlet WPS (duplicate from Fig. 6h) and the local Mexican hat WPS, illustrating the higher frequency resolution obtained with the Morlet wavelet

of $\mathrm{m} / \mathrm{s}$ and $f_{S L}^{\prime}(t)$ in terms of $\mathrm{Hz}$, which govern timecontinuous large-scale variations of amplitude and mean frequency of the small-scales, $u_{S}$, respectively. Linear correlation analyses of these time traces with the largescale velocity, $u_{L}$, are performed in Sect. 4 to study the modulation-type scale-interactions.

\subsection{A note on the instantaneous dissipation}

For turbulent flows we can relate the instantaneous small-scale amplitude and frequency to the rate of turbulent dissipation, $\hat{\epsilon}_{0}(t)$. We here employ a one-dimensiona surrogate, based on the streamwise velocity $u$, following

$\hat{\epsilon}_{0}(t)=15 \nu\left(\frac{\partial u}{\partial x}\right)^{2}$.

An estimate of the mean dissipation scalar is related to the velocity energy spectrum (Pope, 2000). In an instantaneous fashion, for time instant $t_{i}$, an estimate of the instantaneous dissipation, could be obtained in a similar way as $\sigma_{S}\left(t_{i}\right)$ and $f_{S}\left(t_{i}\right)$, via

$\hat{\epsilon}_{0}\left(t_{i}\right)=\hat{\epsilon}+\hat{\epsilon}^{\prime}\left(t_{i}\right)=\frac{1}{\bar{U}^{2}} \int_{0}^{f_{N}} 2 \nu E\left(f, t_{i}\right) f^{2} d f$.

After long-wavelength pass-filtering $\hat{\epsilon}_{0}(t)$, constructed via Eq. (8), we obtain the large-scale variation of the instantaneous dissipation of all scales: $\hat{\epsilon}_{0 L}\left(t_{i}\right)$. The largescale modulation of dissipation can be gleaned from correlating $\hat{\epsilon}_{0 L}\left(t_{i}\right)$ with the concurrent large-scale velocity $u_{L}$. It is worthwhile mentioning that the large-scale variation of dissipation is related to AM and FM (Ganapathisubramani et al, 2012). The reader is referred to
Guala et al (2011) for a set of observations on the modulation of dissipation, computed following Eq. (7).

\section{Modulation results}

The procedure of Sect. 3 resulted in a low-dimensional representation of the time-varying energy spectrum of the small-scales. Here we correlate the constructed time series of large-scale varying amplitude, $\sigma_{S L}^{\prime}$, and mean frequency, $f_{S L}^{\prime}$, with large-scale velocity $u_{L}$ to study $\mathrm{AM}$ and FM, respectively.

\subsection{Modulation coefficients}

We compute the AM coefficient from the small-scale amplitude signal $\sigma_{S L}^{\prime}$, according to

$R_{a}=\frac{\overline{u_{L} \sigma_{S L}^{\prime}}}{\sqrt{\overline{u_{L}^{2}}} \sqrt{\overline{\sigma_{S L}^{\prime 2}}}}$

as opposed to using a large-scale pass-filtered envelope, $E_{L}\left(u_{s}\right)$, of the small-scale $u$ fluctuations, that was employed by Mathis et al (2009) and Talluru et al (2014a). Figure 8a shows a comparison of the coefficients throughout the TBL, computed using $E_{L}$ (denoted as $\left.R_{a}\right)$ and $\sigma_{S L}^{\prime}$ employing the Morlet $\left(R_{a 1}\right)$ and Mexican hat wavelet $\left(R_{a 2}\right)$. The agreement of the three profiles indicate the robustness of the different techniques to represent the small-scale amplitude fluctualions. Coefficients $R_{a 1}$ and $R_{a 2}$ exhibit a slightly stronger zero-time-shift correlation than $R_{a}$, with the Morlet wavelet-based coefficient being the largest $\left(R_{a 1}\right)$. The black solid line indicates an error function profile representing the intermittency $\gamma$, which is defined as the fraction of time that the boundary layer flow is in a turbulent state (Chauhan et al, 2014). Since the boundary layer becomes intermittent for $z / \delta \gtrsim 0.4$, the AM coefficients become strongly negative. This is an inherent effect of the non-turbulent zones that posses a near free stream velocity - and thus a positive large-scale fluctuation - with a low turbulence intensity. Simultaneously, the turbulent bulges in the intermittent region are characterized by a negative large-scale fluctuation with higher small-scale intensity due to the present turbulence. Consequentially, the AM coefficients do not physically represent modulation phenomena in the intermittent region of TBLs, but more likely indicate preferential arrangement (Hutchins, 2014).

We can conveniently extend the definition of the AM coefficient to one that governs FM using the available time-continuous IF signal. And so, we dub this the FM 

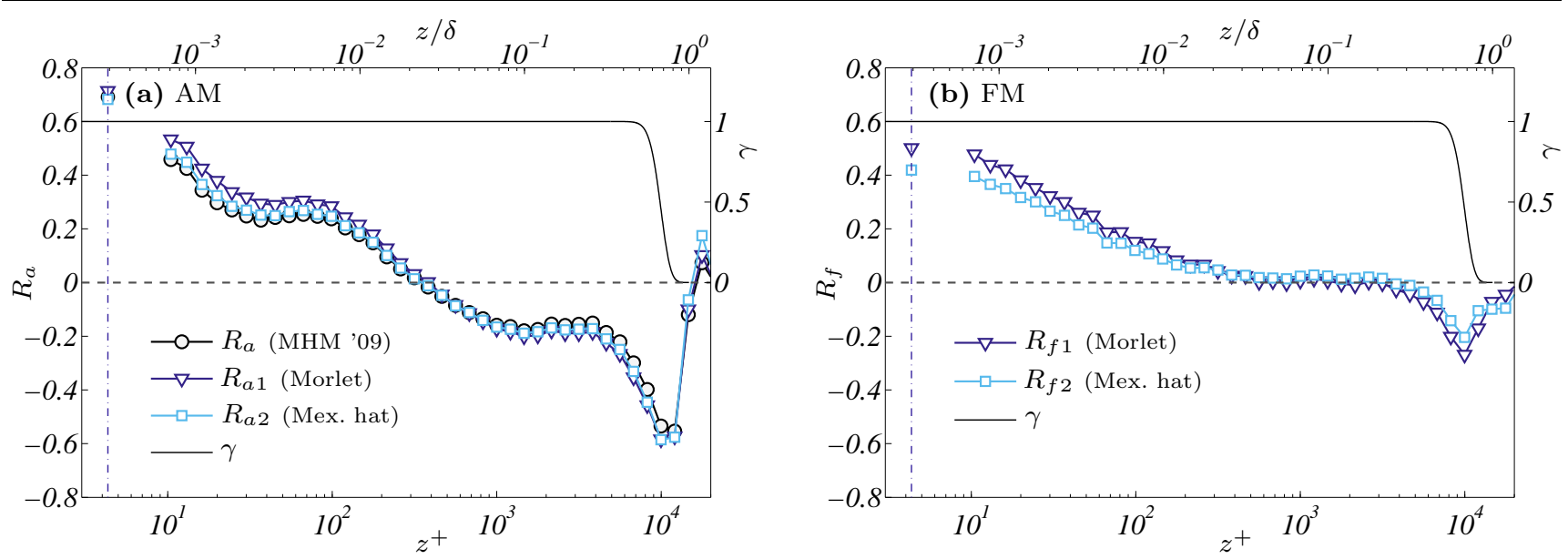

Fig. 8 a Wall-normal evolution of the AM coefficients via Mathis, Hutchins, and Marusic $(2009)\left(R_{a}\right)$ and via the waveletbased approach using the Morlet $\left(R_{a 1}\right)$ and Mexican hat $\left(R_{a 2}\right)$ wavelets. b Evolution of the FM coefficients via wavelet transforms: Morlet $\left(R_{f 1}\right)$ and Mexican hat $\left(R_{f 2}\right)$. Profiles of the intermittency $\gamma$ are shown for reference (solid line)

coefficient, following

$R_{f}=\frac{\overline{u_{L} f_{S L}^{\prime}}}{\sqrt{\overline{u_{L}^{2}}} \sqrt{\overline{f_{S L}^{\prime 2}}}}$.

Profiles of $R_{f}$ are shown in Fig. $8 \mathrm{~b}$ and indicate that the Morlet wavelet-based coefficient, $R_{f 1}$, returns a slightly stronger modulation at zero-time-shift. Furthermore, an absence of FM beyond $z^{+} \approx 400$ is expected, since $R_{f}$ remains zero. However, since the coefficients only provide an insight into the zero-time-shift correlation, this is inconclusive at this stage. Furthermore, no knowledge on temporal shifts within the AM and FM are captured by these coefficients. For these reasons, we consider the isocorrelation maps presented next.

\subsection{Isocorrelation maps of modulation}

Time shifts associated with the AM and FM, at a single position, refer to the relative shift between the largescale velocity and the representative small-scale amplitude or frequency signal, associated with a peak correlation (Fig. 1). Since the shifts vary throughout the TBL (Bandyopadhyay and Hussain, 1984) it is sensible to compute modulation coefficients as a function of time shift, $\tau$, for all wall-normal positions. For AM, the coefficient is given by the inverse Fourier transform of the cross-spectrum $G_{u_{L} \sigma_{S L}^{\prime}}(f)$, via

$R_{a}(\tau)=\frac{\mathcal{F}^{-1}\left[G_{u_{L} \sigma_{S L}^{\prime}}(f)\right]}{\sqrt{\overline{\overline{u_{L}^{2}}}} \sqrt{\overline{\sigma_{S L}^{\prime 2}}}}$.

The cross-correlation coefficient for FM, $R_{f}(\tau)$, can be obtained in a similar manner when $f_{S L}^{\prime}$ is utilized instead of $\sigma_{S L}^{\prime}$. We exclusively consider the temporal correlation coefficient in the current work as this aids in de- termining a single time shift. In contrast, the complexvalued cross-spectra, $G_{u_{L} \sigma_{S L}^{\prime}}(f)$ and $G_{u_{L} f_{S L}^{\prime}}(f)$, include coupled magnitude and phase information, as function of all coherent scales. The magnitude of a similar cross-spectrum for AM was presented by Jacobi and McKeon (2013) who show that most coherence - in the log-region and beyond - is associated with the spectral content of very large scale motions (VLSMs).

Figure 9a shows contours of the cross-correlation coefficient, $R_{a}(\tau)$. Following the notation of Fig. $8 \mathrm{a}, R_{a}$ is computed using the large-scale envelope of the smallscales via the scheme of Mathis et al (2009), while $R_{a 1}$ and $R_{a 2}$ are generated via our wavelet-based time series. Note that a negative shift $(\tau<0)$ implies a lag of the large-scales relative to the small-scales. The trend illustrated by the isocorrelation map agrees with previous studies (Bandyopadhyay and Hussain, 1984; Chung and McKeon, 2010, among others). In close vicinity to the wall, the small-scale amplitude signal leads relative to the large-scales $(\tau<0)$ with a positive correlation. A phase reversal resides in the logarithmic region, meaning that an anti-correlation occurs at an equal but opposite shift. Thus, the small-scale signature lags the large-scales with which they are negatively correlated. Figure 9b shows contours of the FM coefficient $R_{f}(\tau)$. FM is only present in close proximity to the wall $\left(z^{+}<200\right)$ and is modulated in-phase with the AM (Ganapathisubramani et al, 2012). Before discussing the physics embedded in these correlation maps we will consider an accompanying result obtained via conditional averaging. 

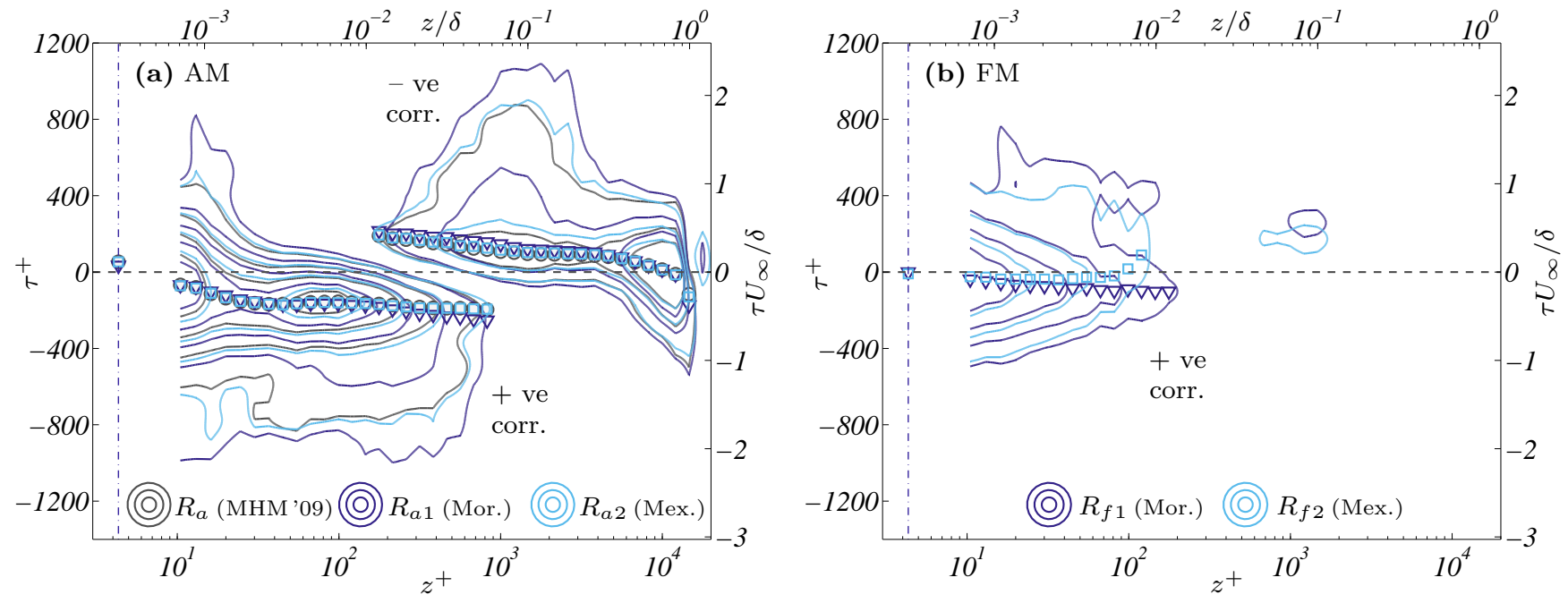

Fig. 9 a Isocorrelation map of the AM coefficient, $R_{a}(\tau, z)$, via Mathis, Hutchins, and Marusic $(2009)\left(R_{a}\right)$ and via the wavelet-based approach using the Morlet $\left(R_{a 1}\right)$ and Mexican hat $\left(R_{a 2}\right)$ wavelets; level range $-0.3-0.5$, step 0.1 (0 contour omitted). b Isocorrelation map of the FM coefficient, $R_{f}(\tau, z)$, via wavelet transforms: Morlet $\left(R_{f 1}\right)$ and Mexican hat $\left(R_{f 2}\right)$; level range 0.1-0.4, step 0.1. A negative time shift $(\tau<0)$ implies a lead of the small-scales relative to large-scales. Extrema of the coefficients at each wall-normal position are identified by the markers, $\circ, \nabla$ and $\square$

\subsection{Conditionally avarged view of modulation}

Previously, Hutchins et al (2011), Talluru et al (2014a), and others, have conditionally averaged large-scale velocity fluctuations, $u_{L}$, alongside the fluctuating smallscale variance, $u_{S}^{2}$. Their condition governed all instants of a negative fluctuation of the large-scale wall-shear stress signal. One of their many findings confirmed that the modulation of the small-scale variance is associated with the large-scale superstructure events. To assist in the physical interpretation of Figs. $9 \mathrm{a}$ and $9 \mathrm{~b}$ we compute conditionally averaged views of the smallscale amplitude and frequency fluctuations. Here we condition the ensemble average on zero-crossings of the large-scale velocity fluctuations at each respective location. This aids in preserving a sufficient temporal resolution in the conditional average, which is otherwise delocalized in time when successive instants of the time series are averaged. In doing so, a variable window averaging technique (as used by Baars et al, 2015) ensured that only the section of the time series between the two zero-crossings, surrounding the conditioning zero-crossing-point, was utilized. Additionally, zero-crossings occurring within $t=2 \delta / \bar{U}$ of each other were excluded from the ensemble to better reveal the dominant features of the scale-interaction; this criterion does not influence our principal conclusions derived from the qualitative magnitude of these conditional averages. Figures $10 \mathrm{a}$ and $10 \mathrm{~b}$ visualize the conditional averages of the large-scale fluctuations of small-scale amplitude, $\sigma_{S L}^{\prime}$, and frequency, $f_{S L}^{\prime}$, respectively. We conditioned on the negative-to-positive zero-crossings of $u_{L}\left(\tau_{\mathrm{np}}^{+}=0\right.$ when $\left.u_{L}=0 \& d u_{L} / d t>0\right)$, shown on the left-hand-side of Figs. 10a and 10b, and the positive-to-negative zero-crossings $\left(\tau_{\mathrm{pn}}^{+}=0\right.$ when $u_{L}=$ $\left.0 \& d u_{L} / d t<0\right)$; any potential breakdown in pointsymmetry (around $u_{L}=0$ ) could be inferred from these results. Finally, the conditional averages employ the Morlet wavelet-based $\sigma_{S L}^{\prime}$ and $f_{S L}^{\prime}$ signals; qualitatively similar results are obtained when averaging the Mexican hat wavelet-based signals.

\subsection{Modulation phenomena and their time shifts}

Hutchins (2014) mentioned that a "fine line" exists between amplitude modulation (near-wall phenomenon) and other features in the TBL that may be better classified as "preferred arrangements" of scales. We here start by describing our conditional views of Figs. 10a and 10b, and the associated isocorrelation maps (Figs. 9a and $9 \mathrm{~b}$ ) in the context of the modulation and preferred arrangement terminology. When concentrating on the domain below the log-region, conservatively $z^{+}<200$, we know that the dynamics near the wall are governed by inner-scaling. The magnitude of the dynamic time scale is $t^{+}=\lambda_{x}^{+} / \bar{U}^{+} \approx 100$ (the inner-spectral peak of the TBL spectogram, Fig. 3b). Moreover, the time scale and fluctuating velocity are fixed in inner units: $t^{+}=t U_{\tau}^{2} / \nu$ and $u^{+}=u / U_{\tau}$. When we preserve time according to a quasi-steady description (Mathis et al, 2013), large-scale motions affect the inner-velocity-scale $\left(U_{\tau} \equiv \sqrt{\tau_{w} / \rho}\right)$ following $\tau_{w}^{\prime}(t) \propto u_{L}(t)$ when first- 
(a) conditionally averaged small-scale amplitude $\sigma_{S L}^{\prime}(\tau) / U_{\tau}$ :

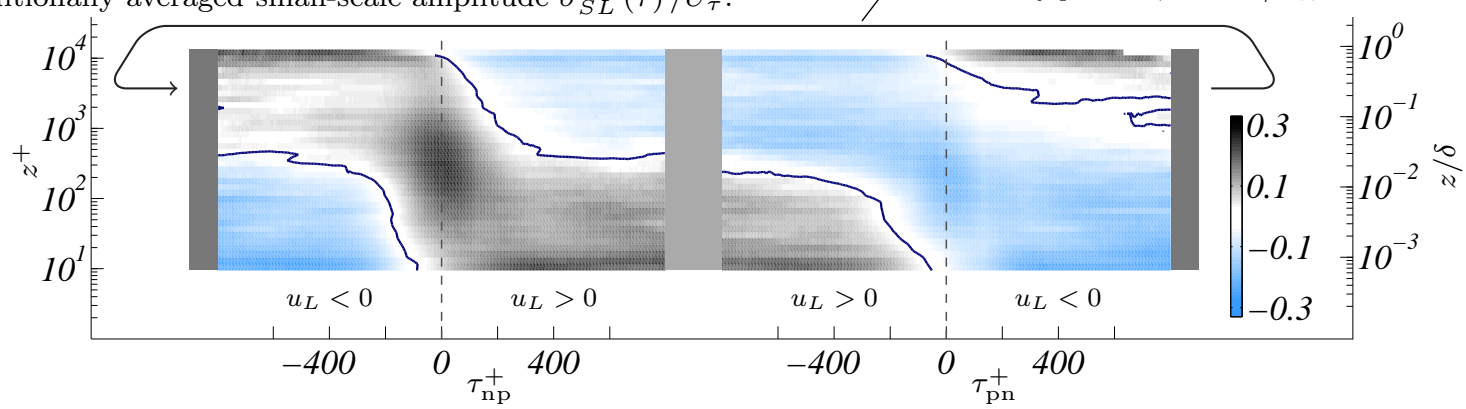

(b) conditionally averaged small-scale frequency $f_{S L}^{\prime}(\tau) \nu / U_{\tau}^{2} \cdot 10^{3}$ :

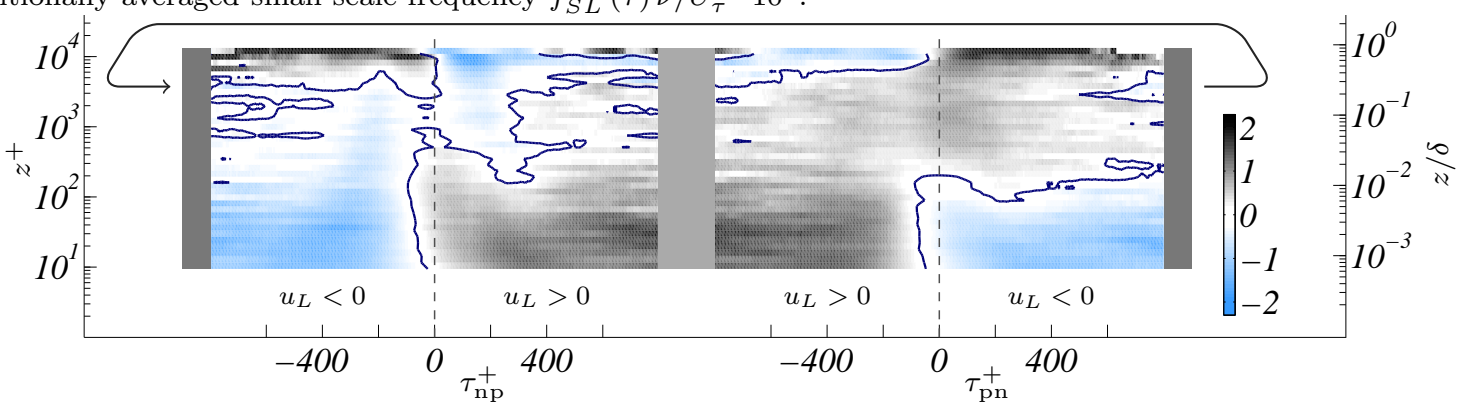

Fig. 10 Conditionally averaged fluctuations of a small-scale amplitude $\sigma_{S L}^{\prime}$, and $\mathbf{b}$ small-scale frequency $f_{S L}^{\prime}$. Fluctuating signals are conditioned on: (1) the negative-to-positive zero-crossings of the local large-scale velocity $\left(\tau_{\mathrm{np}}^{+}=0 \mathrm{when} u_{L}=0 \&\right.$ $\left.d u_{L} / d t>0\right)$, shown on the left-hand-side of the figures, and (2) the positive-to-negative zero-crossings $\left(\tau_{\mathrm{pn}}^{+}=0\right.$ when $u_{L}=0$ $\left.\& d u_{L} / d t<0\right)$, shown on the right-hand-side; the contour line indicates the zero contour

order terms are retained in the analysis; here, $\tau_{w}^{\prime}$ is the fluctuating wall-shear stress. For the inner-scaled small-scale velocity, $u_{S}^{+}$, this implies that any fluctuations increase on an absolute scale when the large-scale velocity exhibits a positive fluctuation $\left(\sigma_{S L}^{\prime}>0\right.$ when $u_{L}>0$ and vice versa); this drives the positive correlation for the AM observed in Figs. 9a and 10a. Similarly, physical time $t$ decreases for a positive fluctuation of the friction velocity, since $t^{+}$of the near-wall cycle is frozen. Hence, the small-scale energy resides at higher frequencies, interpreted as an in-phase FM; this is evidenced by Figs. $9 \mathrm{~b}$ and $10 \mathrm{~b}$ (for $z^{+} \lesssim 200$ ). When we now focus on the intermittent region $(z / \delta \gtrsim 0.4)$ we observe a reversed effect. That is, the amplitude of the smallscales, $\sigma_{S L}^{\prime}$, as well as its representative frequency, $f_{S L}^{\prime}$, exhibit positive fluctuations when the large-scale velocity fluctuations are negative, and vice versa. This is directly caused by an intermittent exposure of the hotwire to turbulent (captured as $u_{L}<0$ ) and non-turbulent fluid $\left(u_{L}>0\right)$. Finally, the arrangement of scales in the log- and wake regions is less well understood, despite over 30 years of compelling research progress on this topic. Bandyopadhyay and Hussain (1984) and Chung and McKeon (2010) describe this region as one comprising a "phase reversal". Hutchins et al (2011) found that the largest fluctuations of the small-scale amplitude, in the log-region, are aligned with the internal shear layers along the inclined back of the low momentum regions (thus around $\tau_{n p}^{+}=0$ ). Fig. 10 provides evidence for the latter in the form of the dark shaded region centered around $\tau_{n p}^{+}=0$ and the center of the log-region $\left(z^{+} \approx 474\right)$.

We now elaborate on the time shifts embedded in the modulation phenomena, which may be contemplated as the relative shifts between $u_{L}$ and the concurrent small-scale representations, $\sigma_{S L}^{\prime}$ and $f_{S L}^{\prime}$, for which peak correlations occur (see the markings in Figs. 9a and 9b). Time shifts are shown in Fig. 11a in our conventional reference frame $\tau$, relative to the large-scales. Note that the time shifts identified from the isocorrelation map of the original AM coefficient (Mathis et al, 2009) are omitted but follow the Morlet wavelet-based shifts; for FM we could only rely on our wavelet-based approach.

Since the large-scale structures are inclined relative to the wall (Fig. 4) we can make a simple coordinate transformation from $\tau$ to a global reference frame $\tau^{\prime}$, relative to the stationary wall. We employ the peak locations, $\tau_{L}$, of the two-point correlation map (Fig. 4), which identified the wall-normal evolution of the time shift associated with the physical inclination of these large-scales; note that $\tau_{L}$ becomes more negative away from the wall (a forward leaning structure). The global view of the time shifts after our coordinate transformation, $\tau^{\prime}=\tau+\tau_{L}$, is presented in Fig. 11b. For time 

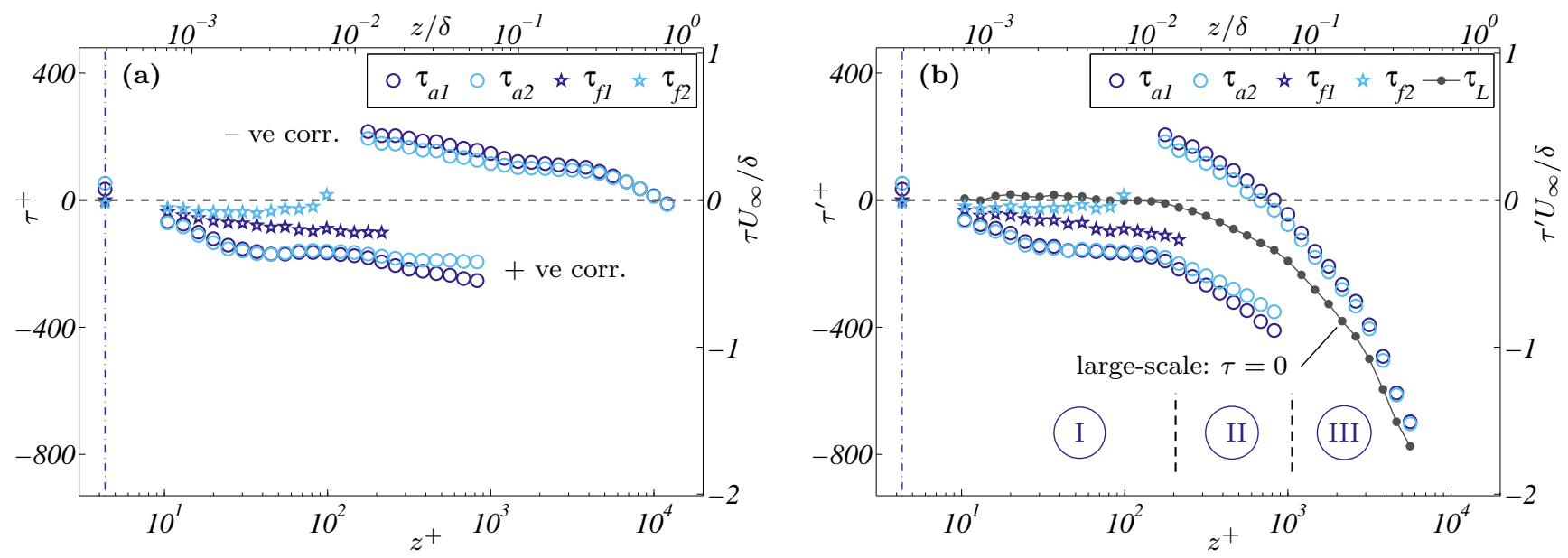

Fig. 11 a Time shifts associated with the extrema in the isocorrelation maps of Figs. 9a and 9b. Here, $\tau_{a}$ and $\tau_{f}$ refer to the time shifts in the AM and FM, respectively. Subscript ' 1 ' indicates use of the Morlet wavelet, and ' 2 ' refers to the procedure utilizing the Mexican hat wavelet. The temporal reference frame $\tau$ can be interpreted as being relative to large-scale structures. b Similar to sub-figure a but for the transformation $\tau \rightarrow \tau^{\prime}$; reference frame $\tau^{\prime}$ is the global frame (Fig. 12)

shifts in $\operatorname{AM}\left(\tau_{a}\right)$ the results for the Morlet $\left(\tau_{a 1}\right)$ and Mexican hat $\left(\tau_{a 2}\right)$ wavelet-based procedure agree reasonably well, particularly when correlations are above 0.2. A larger discrepancy is observed for the FM shift $\left(\tau_{f 1}\right.$ versus $\left.\tau_{f 2}\right)$. In Sects. 3.1 and 3.2 it was shown how the choice of wavelet could eventually affect our constructed signal $f_{S L}^{\prime}$. By employing our chosen wavelets with different temporal and spectral resolutions, it can be argued that the time shift (in a statistical sense) will closely follow the range spanned by $\tau_{f 1}$ and $\tau_{f 2}$. In addition, FM exhibits a lower normalized correlation than AM, which could be a factor in the larger discrepancy, given that peak representation of the broad correlation curve is a crude simplification. Moreover, the magnitude of the time shifts is $\left|\tau^{+}\right|=\mathcal{O}(100)$, which is small relative to the typical time scale at which the large-scale energy resides; the outer-spectral peak in the spectogram (Fig. 3b) exists at $t^{+} \approx 3000$.

We can make several observations from Fig. 11b, although a full explanation of the physics is warranted; this is beyond the current scope as here we focus on an efficient, and time-continuous, procedure to study FM. We have identified three wall-parallel layers in Fig. 11b, denoted as I to III. Layer III encompasses the top part of the log-region (ending at $z^{+}=0.15 R e_{\tau} \approx 2200$ ) and the wake-region above. Only a negative correlation exists between $u_{L}$ and $\sigma_{S L}^{\prime}$ or $f_{S L}^{\prime}$ for the reasons provided earlier. For this preferential scale-arrangement, the small-scale signatures are lagging with respect to the large-scale velocity and blend in with the preferential scale-arrangement in layer II (majority of the log-region), where the phase reversal reflects the arrangement captured in Fig. 10a. Layer I encompasses the wall-normal range below the log-region $\left(z^{+} \lesssim 200\right.$, where AM and FM were shown to be present. The magnitude of the AM shift, $\left|\tau_{a}\right|$, increases progressively with distance from the wall to a near-constant value of $\tau_{a}^{+} \approx-200$ in the range $50 \lesssim z^{+} \lesssim 200$, while the FM shift $\tau_{f}$ is less than half of that. In terms of the normalized modulation strength (Fig. 9a), we observe that the AM is strongest near the wall. In addition, a local maximum in strength is centered around $z^{+} \approx 80$ and coincides with the region where the time shift becomes constant. Interestingly, from the value of $\tau_{L}$ in Fig. 11b we observe that the large-scales remain centered around $\tau^{\prime}=0$ (non-inclined) over this wallnormal range. A summarizing schematic for $z^{+} \lesssim 200$ is depicted in Fig. 12. Imprints of large-scale regions of positive $\left(u_{L}>0\right)$ and negative $\left(u_{L}<0\right)$ fluctuations in close proximity to the wall are indicated in grey-scale. Superposed is a binary signal that represents the largescale structures in space or time, at the wall-normal location of the dashed line. Spatial coordinate $x$ increases to the right, whereas time increases from right-to-left. The modulation physics are visualized in Fig. 12 by a small-scale velocity time series superposed on the largescale binary signal. It is important to note that the envelope of the small scale fluctuations and frequency content are visualized with the crude simplification of having a signature that is periodically similar to the large-scales. Pending research at a range of Reynolds number should address how confined these modulation physics are, in relation to the internal shear layers, and how they scale. Note that Fig. 10, and any other results, are strictly speaking only valid for $R e_{\tau}=14750$. Guala et al (2011) considered a high Reynolds number of $R e_{\tau} \approx 5 \times 10^{5}$ and suggested that the interaction 


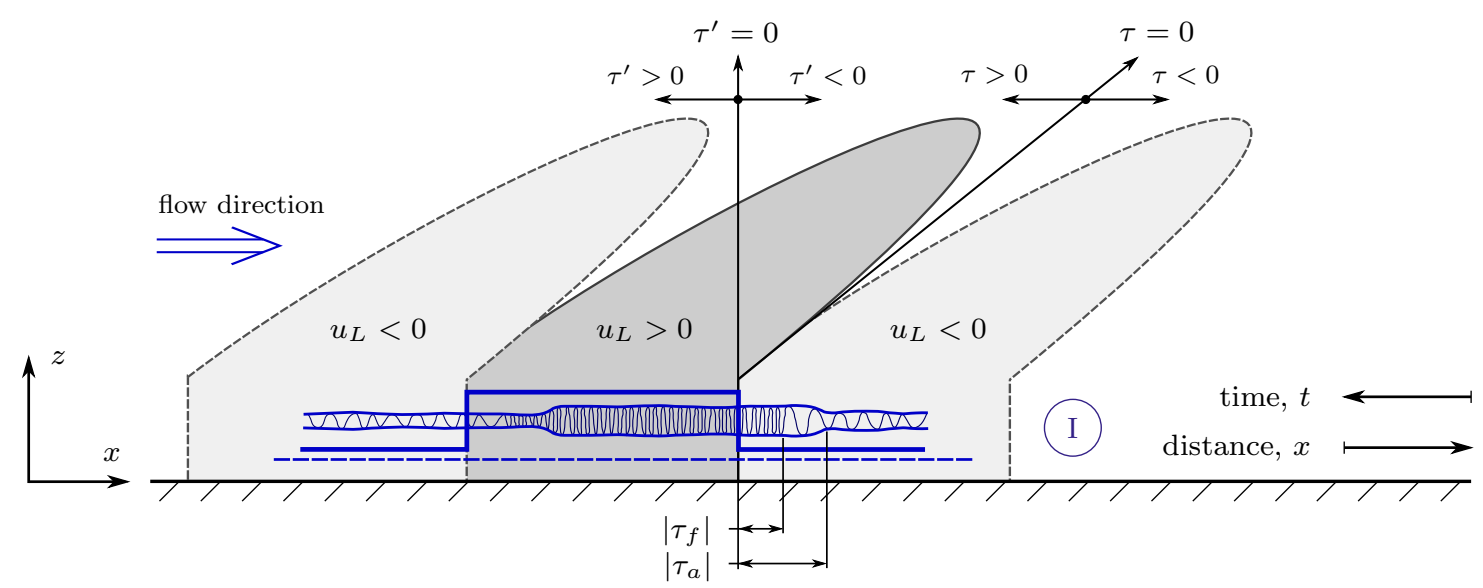

Fig. 12 Conceptual schematic of the AM and FM phenomena in the near-wall buffer layer, relative to the large-scale motions in a turbulent boundary layer with a friction Reynolds number of $R e_{\tau}=14750$

of scales near the wall — and the time shifts - are governed by inner scaling.

Now, we know that in the near-wall region, $\tau_{a}$ is negative and $\left|\tau_{a}\right|>\left|\tau_{f}\right|>0$, meaning that from a stationary observer perspective, an increase in the magnitude of the small-scale fluctuations (at $\tau^{\prime}=\tau_{a}$ ) is noticed first, at least when exclusively considering the streamwise velocity fluctuations. A finite time later, the IF of the small-scales increases (at $\tau^{\prime}=\tau_{f}$ ). Finally, the negative-to-positive zero-crossing of the large-scale streamwise velocity fluctuation passes at $\tau^{\prime}=0$. Hence, the modulated fluctuations in amplitude and frequency lead the large-scale motion (Bandyopadhyay and Hussain, 1984; Guala et al, 2011). Physical mechanisms for this lead demand further research as this could be related to several factors. Our current work is limited to single-point data of the streamwise velocity component alone. Hence, any effects imposed by the threedimensional nature of the large-scale events (Talluru et al, 2014a) and their more complicated footprint cannot be addressed. Dimensions of the regions separating large-scale structures, with positive and negative large-scale velocity fluctuations, are also believed to play an integral role in the explanation of the time shifts we have quantified. These regions are ideally captured by a measure of vorticity, since high-intensity vorticity (larger than the mean vorticity) is concentrated in between the large-scale motions (Klewicki, 2013). Our results, based on the streamwise velocity component, show that the most energetic small-scale fluctuations, in the log-region, are aligned with the internal shear layers along the back of large-scale structures with negative velocity fluctuations. Hypothetically, the time shifts may be the result of a smooth transition between this scale-alignment and its bounding phenomena, being the modulation near the wall and the intermittent behavior near the boundary layer edge, and affiliated bottom-up and top-down influences. This is however speculative and demands further research.

\section{Summary and conclusions}

Investigating amplitude- and frequency-modulations embedded within a broadband signal, such as encountered in the near-wall region of wall-bounded turbulent flows, remains challenging. In this work, we employed a technique to compute amplitude and frequency modulation based on wavelet transforms.

We applied the technique to streamwise velocity fluctuations in a turbulent boundary layer to assess the large-scale modulation of small-scales. The signal's energy was decomposed in time-frequency space to preserve the temporal variations of the spectral energy content, and thereby obtaining its wavelet power spectrum (WPS). Both the Morlet and Mexican hat wavelets are compared for the current application. An inner-scaled separation wavelength of $\lambda_{x}^{+}=7000$ was employed to exclusively consider small-scales. A low-dimensional representation of the small-scale part of the WPS was captured by two new time series. The first one comprises the local standard deviation, obtained by integrating the local WPS in spectral space, while the second signal consists of the instantaneous frequency, computed via the first spectral moment of the local premultiplied spectrum. Large-scale AM and FM were subsequently studied through conventional correlations between the large-scale component of the velocity and the small-scale amplitude and frequency signals, respectively. The advantage of the current procedure is the preservation of time. Statistics of the IF signal could provide new insights into the dynamics occurring in the near-wall region of turbulent boundary layers. It was 
confirmed that the small-scale amplitude signal leads the large-scale signature, while it was found that the lead in frequency modulation is more than a factor of two less. The time shifts were furthermore addressed in the context of the existence of modulation phenomena near the wall, while a preferential arrangement, or alignment of the small-scales relative to the large-scales so to speak, prevails in the log-region and beyond.

Acknowledgements The authors wish to gratefully acknowledge the Australian Research Council for financial support. Furthermore, we would like to give special thanks to Dr. Daniel Chung for insightful discussions.

\section{References}

Addison PS (2002) The illustrated wavelet transform handbook. Taylor \& Francis, New York, NY, USA

Baars WJ, Tinney CE (2013) Transient wall pressures in an overexpanded and large area ratio nozzle. Exp Fluids $54: 1468$

Baars WJ, Ruf JH, Tinney CE (2015) Non-stationary shock motion unsteadiness in an axisymmetric geometry with pressure gradient. Exp Fluids 56(92):1

Bandyopadhyay PR, Hussain AKMF (1984) The coupling between scales in shear flows. Phys Fluids 27(9):2221-2228

Bernardini M, Pirozzoli S (2011) Inner/outer layer interactions in turbulent boundary layers: a refined measure for the large-scale amplitude modulation mechanism. Phys Fluids 23(6), 061701

Blackwelder RF, Kovasznay LSG (1972) Time scales and correlations in a turbulent bounday layer. Phys Fluids 15(9):1545-1554

Boashash B (1992) Estimating and interpreting the instantaneous frequency of a signal - Part 1: fundamentals. Proc IEEE 80(4):520-538

Brown GL, Thomas ASW (1977) Large structure in a turbulent boundary layer. Phys Fluids 20(10):S243-S252

Chauhan K, Philip J, de Silva CM, Hutchins N, Marusic I (2014) The turbulent/non-turbulent interface and entrainment in a boundary layer. J Fluid Mech 742:119-151

Chauhan KA, Monkewitz PA, Nagib HM (2009) Criteria for assessing experiments in zero pressure gradient boundary layers. Fluid Dyn Res 41, 021404

Chung D, McKeon BJ (2010) Large-eddy simulation of largescale structures in long channel flow. J Fluid Mech 661:341364

Cohen L (1989) Time-frequency distributions - a review. Proc IEEE 77(7):941-981

Cohen L (1995) Time-frequency analysis. Prentice-Hall, Inc., Upper Saddle River, NJ, USA

Daubechies I (1992) Ten lectures on wavelets. Soc. Industrial and Applied Math., Philadelphia, PA, USA

Dimotakis PE (2005) Turbulent mixing. Annu Rev Fluid Mech 37:329-356

Duvvuri S, McKeon BJ (2015) Triadic scale interactions in a turbulent boundary layer. J Fluid Mech 767:R4

Farge M (1992) Wavelet transforms and their application to turbulence. Annu Rev Fluid Mech 24:395-457

Favre AJ, Gaviglio JJ, Dumas R (1967) Structure of velocity space-time correlations in a boundary layer. Phys Fluids 10(9):S138-S145
Ganapathisubramani B, Longmire EK, Marusic I (2003) Characteristics of vortex packets in turbulent boundary layers. J Fluid Mech 478:35-46

Ganapathisubramani B, Hutchins N, Monty JP, Chung D, Marusic I (2012) Amplitude and frequency modulation in wall turbulence. J Fluid Mech 712:61-91

Guala M, Metzger M, McKeon BJ (2011) Interactions within the turbulent boundary layer at high Reynolds number. J Fluid Mech 666:573-604

Hutchins N (2014) Large-scale structures in high Reynolds number wall-bounded turbulence. Progress in Turbulence V, Springer Proc Phys 149:75-83, A. Talamelli et al. (eds.)

Hutchins N, Marusic I (2007a) Evidence of very long meandering structures in the logarithmic region of turbulent boundary layers. J Fluid Mech 579:1-28

Hutchins N, Marusic I (2007b) Large-scale influences in nearwall turbulence. Phil Trans R Soc A 365:647-664

Hutchins N, Nickels TB, Marusic I, Chong MS (2009) Hotwire spatial resolution issues in wall-bounded turbulence. J Fluid Mech 635:103-136

Hutchins N, Monty JP, Ganapathisubramani B, Ng HCH, Marusic I (2011) Three-dimensional conditional structure of a high-Reynolds-number turbulent boundary layer. J Fluid Mech 673:255-285

Jacobi I, McKeon BJ (2013) Phase relationships between large and small scales in the turbulent boundary layer. Exp Fluids 54:1481

Klewicki JC (2013) A description of turbulent wall-flow vorticity consistent with mean dynamics. J Fluid Mech 737:176-204

Kulandaivelu V (2011) Evolution and structure of zero pressure gradient turbulent boundary layer. PhD thesis, The University of Melbourne, Dept. of Mechanical Engineering, Melbourne, Australia

Larsson C, Öhlund O (2014) Amplitude modulation of sound from wind turbines under various meteorological conditions. J Acoust Soc Am 135(1):67-73

Ligrani PM, Bradshaw P (1987) Spatial resolution and measurement of turbulence in the viscous sublayer using subminiature hot-wire probes. Exp Fluids 5(6):407-417

Marusic I, Heuer WD (2007) Reynolds number invariance of the structure inclination angle in wall turbulence. Phys Rev Lett 99, 114504

Marusic I, Mathis R, Hutchins N (2010) Predictive model for wall-bounded turbulent flow. Science 329(5988):193-196

Mathis R, Hutchins N, Marusic I (2009) Large-scale amplitude modulation of the small-scale structures in turbulent boundary layers. J Fluid Mech 628:311-337

Mathis R, Marusic I, Chernyshenko SI, Hutchins N (2013) Estimating wall-shear-stress fluctuations given an outer region input. J Fluid Mech 715:163-180

Neuberg J (2000) External modulation of volcanic activity. Geophys J Int 142:232-240

Nickels TB, Marusic I, Hafez S, Chong MS (2005) Evidence of the $k_{1}^{-1}$ law in a high-Reynolds-number turbulent boundary layer. Phys Rev Lett 95, 074501

Pope SB (2000) Turbulent Flows. Cambridge University Press, Cambridge, UK

Rao KN, Narasimha R, Narayanan MAB (1971) The 'bursting' phenomenon in a turbulent boundary layer. J Fluid Mech 48:339-352

Schlatter P, Örlü R (2010) Quantifying the interaction between large and small scales in wall-bounded turbulent flows: A note of caution. Phys Fluids 22

Sreenivasan KR, Prabhu A, Narasimha R (1983) Zerocrossings in turbulent signals. J Fluid Mech 137:251-272 
Talluru KM, Baidya R, Hutchins N, Marusic I (2014a) Amplitude modulation of all three velocity components in turbulent boundary layers. J Fluid Mech 746:R1

Talluru KM, Kulandaivelu V, Hutchins N, Marusic I (2014b) A calibtration technique to correct sensor drift issues in hot-wire anemometry. Meas Sci Technol 105304:1-6
Tomkins CD, Adrian RJ (2003) Spanwise structure and scale growth in turbulent boundary layers. J Fluid Mech 490:3774

Wark CE, Nagib HM (1991) Experimental investigation of coherent structures in turbulent boundary layers. J Fluid Mech 230:183-208 


\section{University Library}

\section{- M M N E R VA A gateway to Melbourne's research publications}

Minerva Access is the Institutional Repository of The University of Melbourne

Author/s:

Baars, WJ;Talluru, KM;Hutchins, N;Marusic, I

Title:

Wavelet analysis of wall turbulence to study large-scale modulation of small scales

Date:

2015-10-01

Citation:

Baars, W. J., Talluru, K. M., Hutchins, N. \& Marusic, I. (2015). Wavelet analysis of wall turbulence to study large-scale modulation of small scales. EXPERIMENTS IN FLUIDS, 56 (10), https://doi.org/10.1007/s00348-015-2058-8.

Persistent Link:

http://hdl.handle.net/11343/282540 